\title{
Malignant Hyperthermia Syndrome
}

National Cancer Institute

\section{Source}

National Cancer Institute. Malignant Hyperthermia Syndrome. NCI Thesaurus. Code C84869.

A rare disorder characterized by rapid rise of the body temperature, accompanied by rhabdomyolysis and, if untreated, by collapse and death. It occurs in susceptible individuals who receive certain drugs for general anesthesia, gas anesthetics, or succinylcholine. It may be inherited in an autosomal dominant pattern. 